\title{
Évolution des parois rocheuses gelées de haute montagne sous forçage climatique
}

\author{
Ludovic Ravanel, Florence Magnin, Xavi Gallach, Philip Deline \\ Laboratoire Edytem (Environnements, dynamiques et territoires de montagne), \\ Université Savoie Mont-Blanc / CNRS, Chambéry
}

ludovic.ravanel@univ-smb.fr

\section{Résumé}

Avec le réchauffement du climat, la dégradation du permafrost est à l'origine d'une intensification des processus géomorphologiques sur les versants de haute montagne. Dans les parois rocheuses, les écroulements se multiplient et leur volume augmente, posant des problèmes de sécurité non seulement à haute altitude (infrastructures, alpinistes), mais également pour les fonds de vallée. Cet article présente les travaux récemment menés dans le massif du MontBlanc sur la relation entre climat et écroulements à différentes échelles de temps, les effets des épisodes caniculaires et la répartition et l'évolution du permafrost de paroi.
D ans la haute montagne alpine, les pentes longues et raides des versants et la présence d'importants volumes rocheux amplifient les processus géomorphologiques (avalanches, laves torrentielles, coulées boueuses, etc.). Du fait de l'existence d'un étagement bioclimatique, ces processus se succèdent le long des versants, voire s'y combinent pour affecter non seulement ceux-ci mais également les fonds de vallée. En partie constitué de parois rocheuses raides, de temps à autre couvert de glace mobile ou immobile, le versant est protéiforme, changeant dans le temps et dans l'espace. Parfois, des pans entiers de versant se déstabilisent, comme récemment au Piz Cengalo (3 $369 \mathrm{~m}$, massif de la Bernina). Au voisinage du célèbre Piz Badile (3 308 m) et du Piz Bernina (4 $049 \mathrm{~m})$ - point culminant des Alpes orientales -, ses faces nord-est et nord-ouest dominent le territoire suisse de Bregaglia, dans le canton des Grisons. Il a été plusieurs fois sujet à des écroulements (de volume supérieur à $100 \mathrm{~m}^{3}$ ), voire à des avalanches rocheuses (de volume supérieur à 1 million de mètres cubes ou $\mathrm{Mm}^{3}$ ), comme en décembre 2011 lorsqu'environ $1,5 \mathrm{Mm}^{3}$ de roche s'en sont détachés avant de recouvrir un alpage. D'autres écroulements moins volumineux ont suivi en 2012, 2016 et le 21 août 2017. Le 23 août 2017, une avalanche rocheuse d'un volume estimé à $3,1 \mathrm{Mm}^{3}$ a engendré une coulée boueuse qui a atteint le village de Bondo (figure 1 ; Walter et al., 2020), heureusement préalablement évacué grâce à un système d'alerte installé après l'événement de 2011. Huit alpinistes et randonneurs ont toutefois perdu la vie.
$\mathrm{Au}$ regard d'événements de ce type, il est impératif de mieux comprendre l'évolution des versants et en particulier des parois de haute montagne pour mieux anticiper les risques directs et indirects associés aux processus qui les affectent. D'autant que la fréquence des écroulements semble augmenter depuis trois décennies, en particulier depuis l'été 2003 qui est resté dans les mémoires comme une saison très défavorable à la pratique de l'alpinisme, en raison notamment des très nombreux éboulements (de volume inférieur à $100 \mathrm{~m}^{3}$ ) et écroulements rocheux qui l'ont émaillée en haute montagne (Gruber et al., 2004). Cet été-là a provoqué une prise de conscience des changements en cours dans la haute montagne alpine chez les alpinistes comme dans le monde académique, renforçant l'hypothèse d'une relation étroite entre la dégradation du permafrost - c'est-à-dire le réchauffement des terrains (matériaux lithosphériques) gelés en permanence - et la déstabilisation des versants de haute montagne.

Comparativement au permafrost circum-arctique qui caractérise de très vastes espaces et pour lequel les premiers travaux systématiques de recherche remontent aux années 1940 du fait des implications sur la construction d'infrastructures (oléoducs, gazoducs, mines, installations militaires, etc.), le permafrost alpin ne fait l'objet d'études approfondies que depuis les années 1970 avec les premiers travaux réalisés en Suisse. Les premières recherches sur le permafrost des parois rocheuses sont plus récentes encore, d'abord motivées 


\section{Abstract}

Frozen high-mountain rock walls response to climate change

Under global warming, permafrost degradation tends to intensify geomorphological processes on high mountain slopes. In the perennially frozen rock walls, the number and volume of rockfalls is increasing, causing safety problems not only at high altitude (infrastructure, mountaineers) but also for the valleys. This article summarizes recent work carried out in the Mont-Blanc massif on the climate-rockfall relationship at different time scales, the effects of heat waves, and the distribution/evolution of rock wall permafrost.
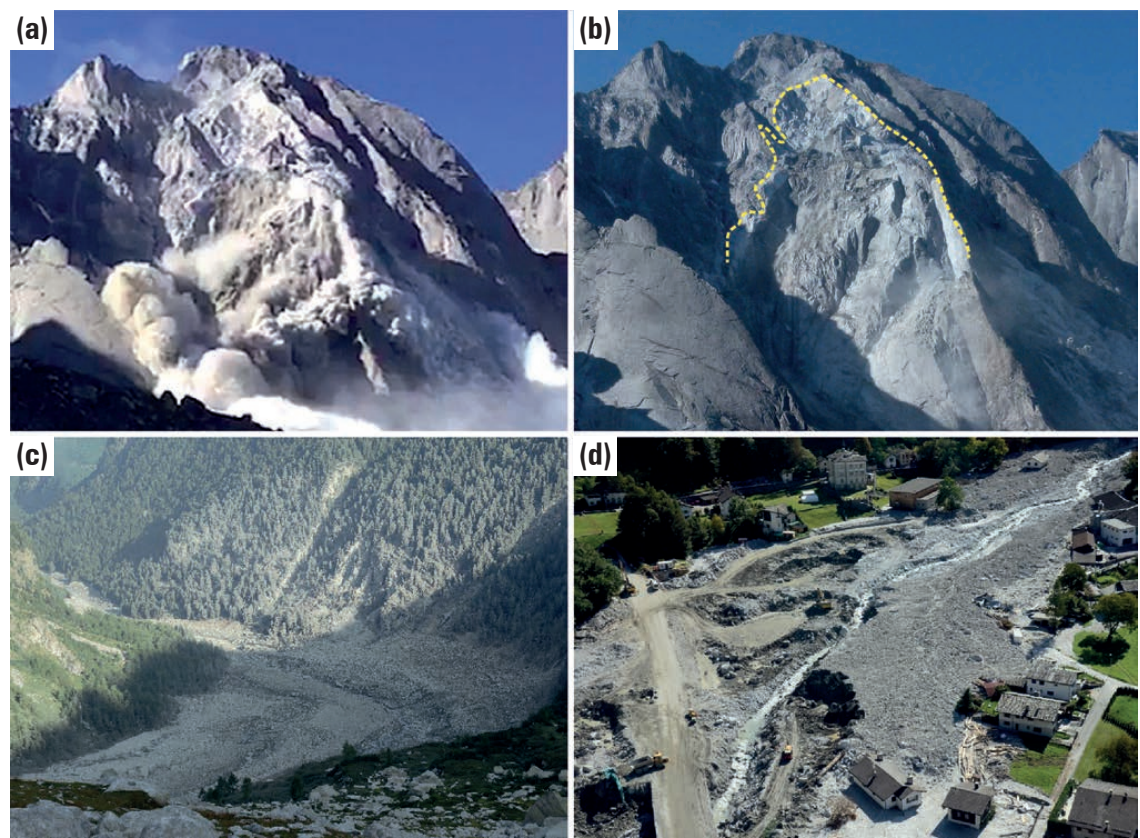

Figure 1. L'avalanche rocheuse du Piz Cengalo (3 369 m) du 23 août 2017 (massif de la Bernina, Suisse). (a) Avalanche rocheuse de 3,1 $\mathrm{Mm}^{3}$ sur le glacier Vadrec dal Cengal. En bas, le nuage blanc résulte de l'impact subi par un petit glacier pulvérisé au pied de la paroi rocheuse (photo : Robert Salis). (b) Photo post-écroulement du versant nord-est du Piz Cengalo (source : Institut für Schneeund Lawinenforschung, Davos) ; en tiretés, les limites de la cicatrice d'écroulement. (c) L'avalanche rocheuse a dévalé le fond du val Bondasca (photo : Marcia Phillips). (d) Le village de Bondo (altitude : $800 \mathrm{~m}$ ) après le passage des coulées boueuses ; une centaine de bâtiments ont été détruits ou très endommagés (photo : Marcia Phillips).

par la construction d'infrastructures avec des problèmes de stabilité liés au permafrost (Haeberli et al., 1997). À la différence du permafrost des hautes latitudes, le permafrost alpin est fortement contraint par la topographie, la micro-climatologie, le matériel de subsurface, et les couvertures nivales et glaciaires. C'est pourquoi mesurer et suivre la répartition du permafrost de paroi et la modéliser est un champ de recherche en tant que tel. À côté de ces travaux, une large place a été faite à l'étude des déstabilisations rocheuses associées à la dégradation du permafrost, avec pour postulat que la glace présente dans les fractures des parois à permafrost joue le rôle de ciment et y empêche la circulation d'eau (Gruber et Haeberli, 2007). Cet article présente quelques-uns de nos travaux menés dans le massif du Mont-Blanc, notamment ceux visant à vérifier la relation entre climat et écroulements à l'échelle du dernier siècle et demi comme à celle des 20 derniers millénaires. Afin de vérifier que cette relation repose effectivement sur la dégradation du permafrost, nous avons étudié les écroulements recensés de manière exhaustive dans le massif depuis plus d'une décennie, en particulier lors de l'été caniculaire 2003. Cette approche nous a conduits à étudier la répartition du permafrost à l'échelle du massif et à en modéliser l'évolution dans plusieurs parois.

\section{Le massif du Mont-Blanc comme laboratoire des parois rocheuses de haute altitude}

Le massif du Mont-Blanc se situe sur la marge occidentale de la chaîne alpine. Avec ses $550 \mathrm{~km}^{2}$ environ, il s'étend sur trois pays : France (Haute-Savoie et Savoie), Italie (vallée d'Aoste) et Suisse (Valais). Parmi les montagnes occidentales, aucune n’a été aussi visitée, décrite et citée en exemple (Bozonnet et al., 1983). En glaciologie et en géologie notamment, c'est le plus étudié des massifs cristallins des Alpes externes. La quasi-totalité des roches qui le composent est cristalline, le granite du mont Blanc étant intrusif dans une série métamorphique hercynienne. Si le mont Blanc culmine à $4809 \mathrm{~m}$, nombre de sommets et faces granitiques du massif s'élèvent bien au-delà de $3000 \mathrm{~m}$ d'altitude, dont 28 des 82 sommets des Alpes supérieurs à $4000 \mathrm{~m}$ (figure 2). La plupart de ces derniers composent la ligne de partage des eaux entre les bassins du Rhône et du Pô. Le massif se caractérise par un englacement sur environ $25-30 \%$ de sa surface $\left(140 \mathrm{~km}^{2}\right.$ une superficie supérieure à $5 \mathrm{~km}^{2}$. de glaciers) et 12 de ses 94 glaciers ont 


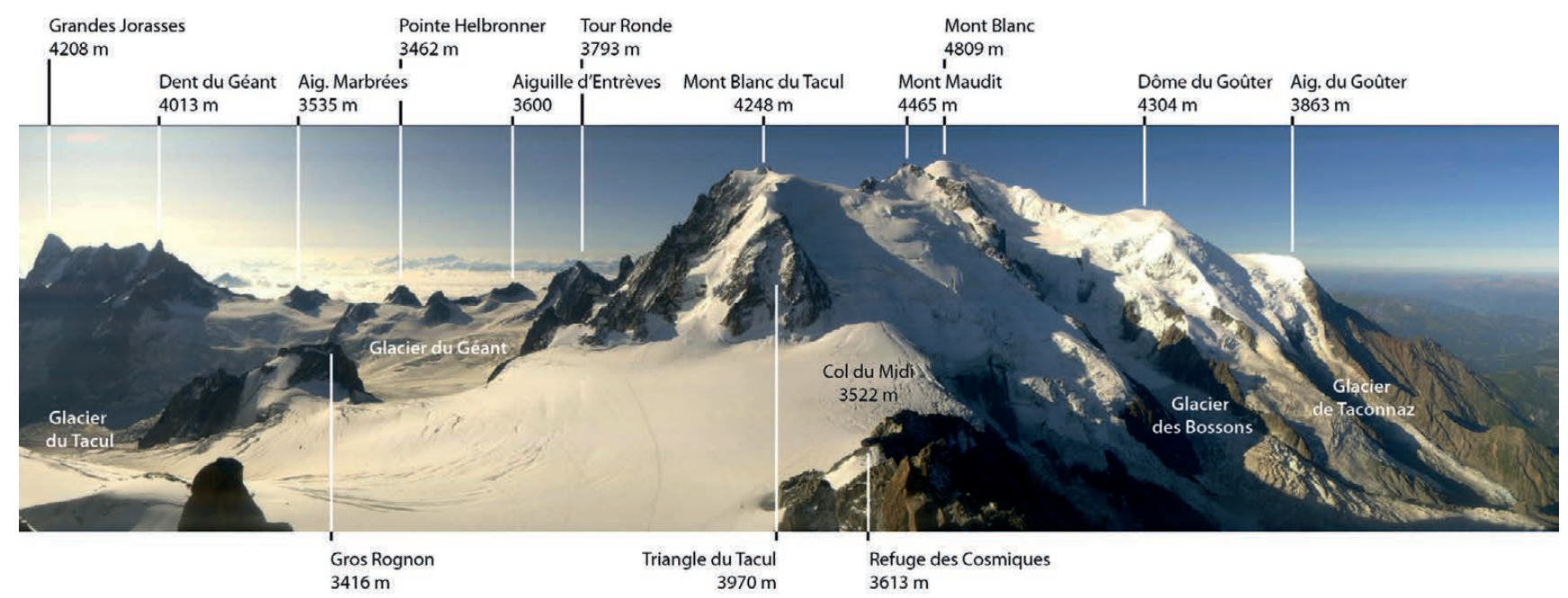

Figure 2. Le massif du Mont-Blanc (partie sud-ouest) vu depuis l'aiguille du Midi (3 842 m). Photo : Panocam CMB, 24 août 2019.

Avant les premiers travaux sur les parois rocheuses du massif du MontBlanc, plusieurs glaciers ont été étudiés du point de vue de leurs interactions avec les avalanches rocheuses qui ont affecté les versants qui les encadrent (Deline, 2009), souvent sur le versant italien. Celui-ci constitue un terrain d'étude exemplaire sur ce thème tant la dénivellation et la raideur de ses versants exacerbent l'effet de dominance et perturbent l'étagement morphodynamique. Le glacier de la Brenva, dont la dynamique a été modifiée par de nombreuses avalanches rocheuses au cours de l'Holocène, dont deux lors du $\mathrm{XX}^{\mathrm{e}}$ siècle (figure 3), a notamment été étudié. Dans le Val Ferret, la grande avalanche rocheuse du Triolet de 1717 met en évidence l'impact géomorphologique considérable que peuvent avoir les grandes déstabilisations rocheuses dans la haute montagne englacée.

Si l'englacement est généralement une caractéristique de la haute montagne alpine, celle-ci peut également présenter un vaste domaine périglaciaire (caractérisé par l'alternance de phases liquide et solide de l'eau consécutive aux variations de température), voire supraglaciaire. Au-dessus de la limite de la neige permanente, la probabilité que le versant soit couvert par un glacier est forte. Néanmoins, de vastes surfaces rocheuses sont présentes au-delà de cette limite pour des raisons topographiques et structurales (raideur, topographie en éperon et pilier, etc.). L'étage supraglaciaire est donc quasi exclusivement constitué de parois rocheuses raides, caractérisées par une certaine " immunité » géomorphologique. En effet, elles n'ont pas (ou peu) subi d'érosion glaciaire ni d'actives alternances gel/ dégel et peuvent comporter - en particulier celles exposées au nord - des couvertures de glace stabilisatrices, " cimentant » les volumes rocheux sous-jacents. Aujourd'hui, le changement climatique en cours remet largement en question cette immunité.

\section{Le lien entre climat et écroulements à différentes échelles de temps}

Le lien entre climat et écroulements a d'abord été étudié sur la période post-Petit Âge Glaciaire pour l'emblématique face ouest des Drus (3 $754 \mathrm{~m}$ ).
L'attrait touristique de cette paroi presque verticale d'une dénivellation de $1000 \mathrm{~m}$ a engendré une iconographie abondante, permettant de comparer un grand nombre de photographies précisément datées. Il est à noter que la photographie de montagne, née vers 1850, est concomitante de la fin du Petit Âge Glaciaire (PAG). L'interprétation de ces photographies, complétée par des témoignages historiques et un modèle numérique de terrain à haute résolution acquis par balayage laser terrestre, a permis une reconstitution de l'évolution de l'instabilité de la face ouest des Drus sur 150 ans (Ravanel et Deline, 2008). Huit écroulements rocheux ont arraché à cette face, entre 1905 et 2005 , un volume total d'environ $335000 \mathrm{~m}^{3}$. Leur enchaînement

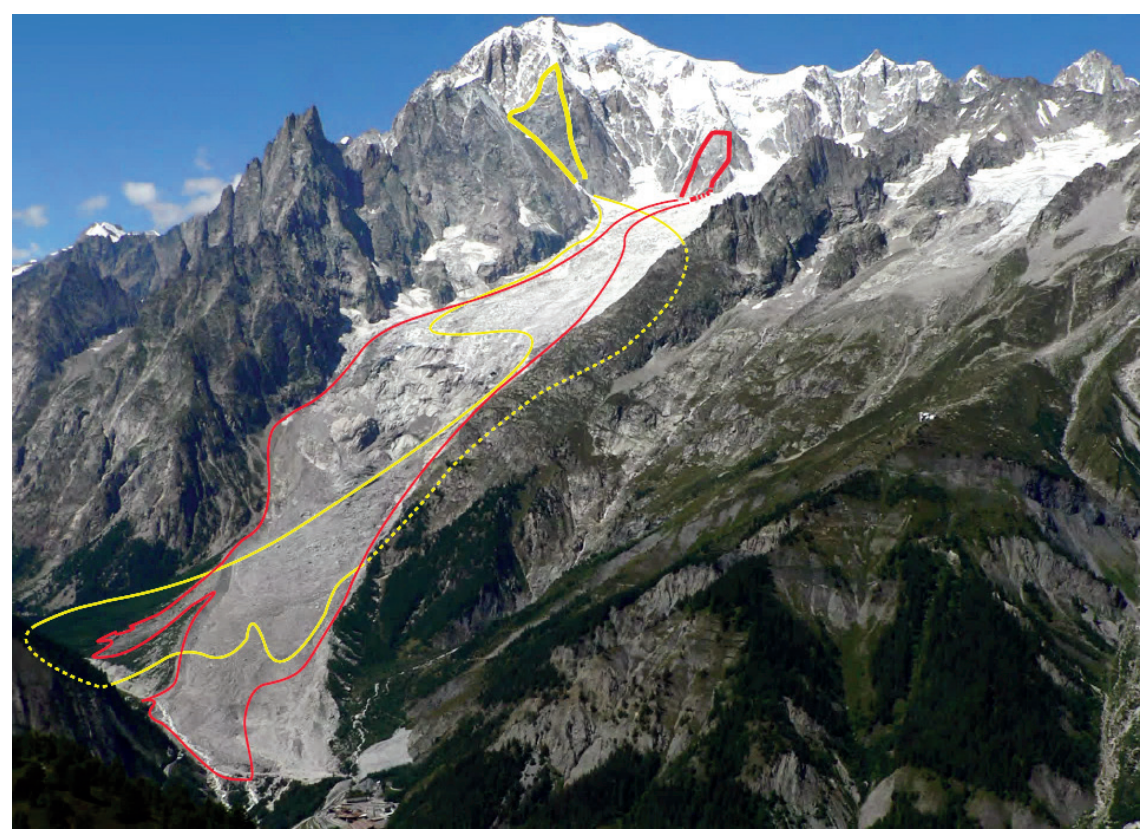

Figure 3. Le glacier de la Brenva en 2005 (Deline, 2009) avec les cicatrices (traits plus épais, en haut) et les emprises (traits fins, discontinus dans les secteurs masqués) des avalanches rocheuses des 19 novembre 1920 (en jaune) et 18 janvier 1997 (en rouge), issues respectivement du Grand Pilier d'Angle (4 $243 \mathrm{~m}$ ) et de l'Éperon de la Brenva (3 $725 \mathrm{~m}$ ). Le point culminant est le sommet du mont Blanc (4 809 m). La sortie du tunnel du Mont-Blanc (1 $370 \mathrm{~m}$ ) est visible au bas de la photo. 
a produit l'érosion régressive (du bas vers le haut) du pilier Bonatti, qui s'est accélérée à partir de 1950 avec des volumes et une fréquence croissants jusqu'à la disparition complète du pilier lors de l'écroulement de juin 2005 $\left(292000 \mathrm{~m}^{3}\right.$ ), de manière concomitante avec l'élévation de la température de l'air dans ce secteur des Alpes. Pour conforter ces résultats, un travail similaire a concerné l'ensemble du versant nord des aiguilles de Chamonix qui se développe sur $5 \mathrm{~km}$ au-dessus de la vallée. Les parois rocheuses n'y ont pas connu de changements notables entre la fin du Petit Âge glaciaire et la fin des années 1940 : les deux premiers écroulements ne se sont produits qu'en 1947, sur la large face ouest de l'aiguille de Blaitière (3 $514 \mathrm{~m}$ ).

Les 67 écroulements de 500 à $65000 \mathrm{~m}^{3}$ ainsi documentés depuis 1862 présentent, comme pour les Drus, une très bonne corrélation avec les périodes les plus chaudes (Ravanel et Deline, 2011). Ainsi, $82 \%$ des écroulements se sont produits au cours de la période 19902015, caractérisée par l'accélération du réchauffement climatique, et les étés les plus chauds (1947, 1976, 1983, 2003, 2015) ont été très favorables au déclenchement d'écroulements, avec une fréquence maximale lors de l'été caniculaire de 2003. La mise en évidence de l'influence du climat sur les écroulements (figure 4) a validé l'hypothèse de Wilfried Haeberli (université de Zurich, Suisse), développée à partir des années 1970, d'une étroite relation entre instabilités de versant (de parois rocheuses ici) et réchauffement climatique. Le rôle prépondérant de la dégradation du permafrost sur la déstabilisation des parois de haute montagne a également été souligné. En effet, i) l'ensemble des écroulements s'est produit en contexte de permafrost d'après les modèles disponibles de sa répartition ; ii) de nombreuses cicatrices d'arrachement observées à partir de 2007 contenaient de la glace ; iii) l'altitude moyenne des cicatrices (3 $130 \mathrm{~m}$ ) est proche de la limite inférieure modélisée du permafrost, où sa dégradation est la plus active ; iv) les écroulements affectent préférentiellement les crêtes, éperons et piliers, dont la topographie accélère la dégradation du permafrost en raison de la propagation des flux de chaleur latéraux depuis les secteurs exposés au rayonnement solaire ; v) l'été caniculaire de 2003 n'a connu ni fortes précipitations ni séisme ; vi) rares sont les écroulements qui peuvent s'expliquer par la décompression des parois suite au retrait des glaciers (absence d'un glacier à proximité).
Les observations des Drus et des aiguilles de Chamonix, basées sur des photographies et complétées avec les observations plus récentes sont limitées au dernier siècle et demi. Il a fallu dépasser cette limite temporelle pour étudier la relation entre climat et écroulements pendant le Tardiglaciaire et l'Holocène - c'est-à-dire sur environ 20000 ans. Cela a été possible en se fondant sur la datation de nombreuses cicatrices d'écroulement dans le massif par la méthode des isotopes cosmogéniques. Ce sont ainsi 80 échantillons de granite qui ont été prélevés sur neuf parois. Ils ont permis de dater 62 écroulements dont les âges sont compris entre 300 ans et $88,4 \pm 7,6 \mathrm{ka}$ BP. L'analyse statistique des âges holocènes a mis en évidence quatre (a)

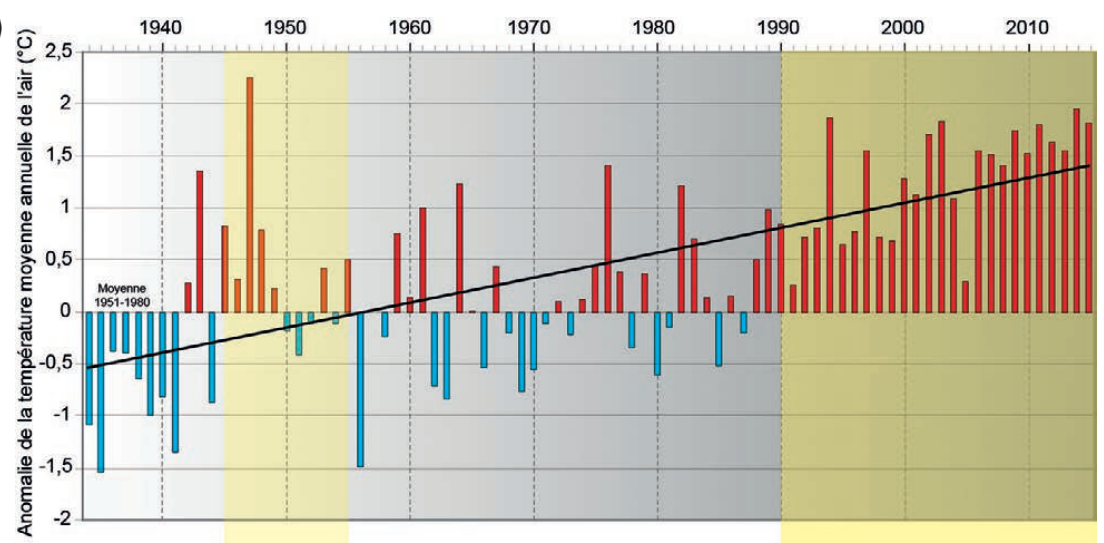

(b)

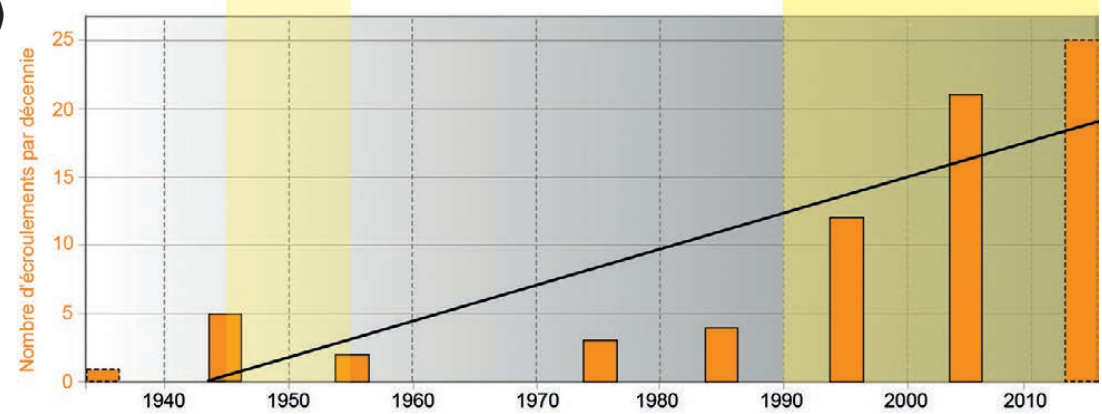

Figure 4. Température moyenne annuelle de l'air à Chamonix (1 040 m) de 1934 à 2015 et nombre d'écroulements (de volume supérieur à $100 \mathrm{~m}^{3}$ ) par décennie dans la face ouest des Drus et sur le versant nord des aiguilles de Chamonix. Droites : régressions linéaires. NB : la dernière période ne couvre que cinq ans (2011-2015).

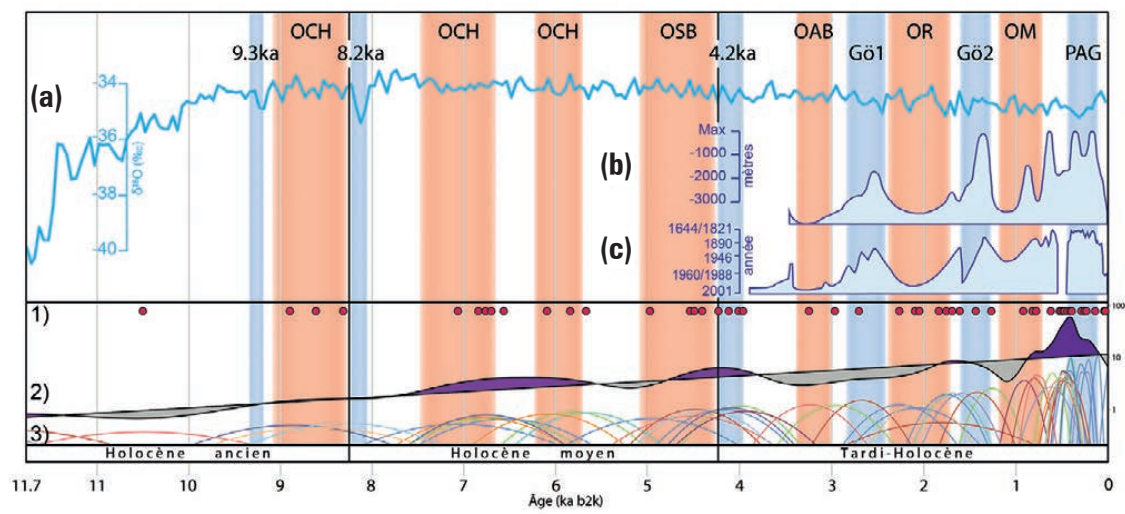

Figure 5. Âges des écroulements holocènes et variables associées à la température de l'air pendant I'Holocène (Gallach et al., 2020, modifié). (a) Profil isotopique NGRIP ; (b) variations du front du grand glacier d'Aletsch depuis 1400 BC par rapport à son extension en 2000 ; (c) variations altitudinales de la Mer de Glace sous le site touristique du Montenvers. Périodes climatiques holocènes : 9,3 ka : événement 9,3 ka ; OCH : optima chauds de l'Holocène ; 8,2 ka : événement 8,2 ka ; 0SB : optimum sub-boréal ; 4,2 ka : avancée 4,2 ka ; OAB : optimum de l’Âge de Bronze ; Gö1 : Göschener 1 ; OR : optimum romain ; Gö2 : Göschener 2 ; OM : optimum médiéval ; PAG : Petit Âge Glaciaire ; 1) âges moyens (sans les incertitudes associées) des écroulements holocènes ; 2) distribution statistique, selon les âges et leurs incertitudes, de la probabilité d'écroulement (nombre d'écroulements par siècle) au cours de l'Holocène autour d'une régression linéaire ; 3) représentation des âges et incertitudes des écroulements. Échelle à droite : probabilité d'écroulement (en nombre d'écroulements/100 ans). 
périodes d'occurrence accrue d'écroulements (figure 5) : i) vers 7,0-5,7 ka, correspondant aux optima chauds de l'Holocène ; ii) 4,5-4,0 ka, coïncidant avec l'optimum sub-boréal ; iii) vers 2,3-1,6 ka, lié à l'optimum climatique romain; et iv) vers $0,9-0,3 \mathrm{ka}$, période correspondant à l'optimum climatique médiéval et au début du Petit Âge glaciaire (Gallach et al., 2020). Ces résultats confirment, ici sur un pas de temps long, l'influence du climat sur le déclenchement des écroulements.

\section{Les étés caniculaires, accélérateurs de la morphodynamique}

L'année 2003, avec plusieurs longs épisodes caniculaires, a permis une prise de conscience à la fois du réchauffement climatique et des conséquences possibles de celui-ci, notamment en haute montagne. Dans la région du mont Blanc, la seconde quinzaine du mois d'avril a vu le printemps arriver subitement, avec des températures élevées pour la saison. Durant le mois de mai, les températures ont continué à augmenter. En juin, des records de durée d'ensoleillement et de chaleur ont été enregistrés. En juillet, après un court répit en début de mois, la canicule s'est imposée de nouveau. La fin de mois est restée très chaude, ponctuée d'orages. En août, la canicule s'est poursuivie jusqu'à l'épisode orageux du 28 au 31 août. Finalement, l'été 2003 fut le plus chaud jamais enregistré par les stations météorologiques à Chamonix, dans les Alpes et en Europe. Dans le massif du Mont-Blanc, entre le $1^{\text {er }}$ juin et le 31 août 2003 , l'isotherme $0{ }^{\circ} \mathrm{C}$ s'est élevé au-dessus de $3500 \mathrm{~m}$ d'altitude pendant 83 jours, dépassant même $4800 \mathrm{~m}$ le 2 août. Début août, pendant 16 jours, la température est restée positive (y compris la nuit) à l'aiguille du Midi (3 $842 \mathrm{~m}$ ). Malgré le très beau temps qui a perduré toute la saison, l'été 2003 est resté dans les mémoires comme très défavorable à la pratique de l'alpinisme. En effet, la température élevée a empêché le regel nocturne de la neige, les rimayes (crevasses situées entre les couvertures de glace immobiles ou les parois rocheuses et les glaciers mobiles) sont devenues infranchissables et les écroulements rocheux se sont multipliés, rendant les itinéraires rocheux dangereux. Ces écroulements ont été recensés sur les glaciers du massif du Mont-Blanc à l'aide d'une image prise par le satellite Spot-5 le 23 août (Ravanel et al., 2017). Les caractéristiques des écroulements ont été établies en recourant à des modèles numériques de terrain, à des cartes et à des estimations (épaisseur des dépôts, par exemple).

Cet été-là, 182 écroulements (ou séries résulter de plusieurs écroulements) ont été documentés (Ravanel et al., 2017). Ils sont répartis de manière homogène dans le massif, avec trois secteurs plus affectés : celui du mont Blanc du Tacul (4 $248 \mathrm{~m}$ ), des aiguilles de Chamonix et de l'ensemble aiguille Verte-Droites-Courtes (figure 6). La répartition des écroulements en fonction de l'altitude de la cicatrice est assez régulière : leur nombre croît de 2900 à 3400 m d'altitude, la tranche altitudinale la plus affectée étant à 3 400-3 $500 \mathrm{~m}$, avec 37 écroulements (20\%). Le nombre d'écroulements décroît ensuite régulièrement jusqu'à $4200 \mathrm{~m}$. Un seul écroulement a été recensé en dessous de $3000 \mathrm{~m}$ d'altitude. $65 \%$ des écroulements ont un volume compris entre 100 et $1000 \mathrm{~m}^{3}$, $13 \%$ entre 1000 et $2000 \mathrm{~m}^{3}$, et $18 \%$ entre 2000 et $10000 \mathrm{~m}^{3}$ (volume moyen : $1800 \mathrm{~m}^{3}$; volume total : $340000 \pm 100000 \mathrm{~m}^{3}$ ). L'écroulement principal, dans la face ouest de l'aiguille du Plan, était de $30000 \mathrm{~m}^{3}$. Les écroulements sont pour la plupart superficiels (quelques mètres d'épaisseur) et seuls six d'entre eux ont affecté une paroi à température modélisée faiblement positive (voir ciaprès). Avec d'autres indices (répartition altitudinale des écroulements, d'écroulements, car un dépôt pouvait

glace observée dans de nombreuses cicatrices, absence d'autres facteurs explicatifs, etc.), ceci suggère le rôle très probable de la dégradation du permafrost dans le déclenchement des écroulements de 2003 dans le massif du Mont-Blanc - un rôle confirmé par l'analyse des écroulements d'un autre été caniculaire, celui de 2015 (Ravanel et al., 2017).

\section{Une question de permafrost}

Le permafrost correspond à tout matériel lithosphérique (sol, roche en place, formations superficielles) dont la température reste durablement négative avec présence ou non de glace. Dans la plupart des cas, le permafrost a plusieurs milliers d'années voire davantage. Sa dégradation peut favoriser différents processus géomorphologiques en fonction du type de terrain, du degré de pente et du contenu en glace. Dans les formations superficielles de haute montagne (éboulis, moraines ou glaciers rocheux), la dégradation du permafrost peut aboutir à des phénomènes rapides et préjudiciables pour les implantations humaines (accélération du fluage des masses gelées, déstabilisation des glaciers rocheux, apport de sédiments aux laves torrentielles, etc.).

La compréhension du rôle de la dégradation du permafrost dans le déclenchement d'écroulements (Krautblatter

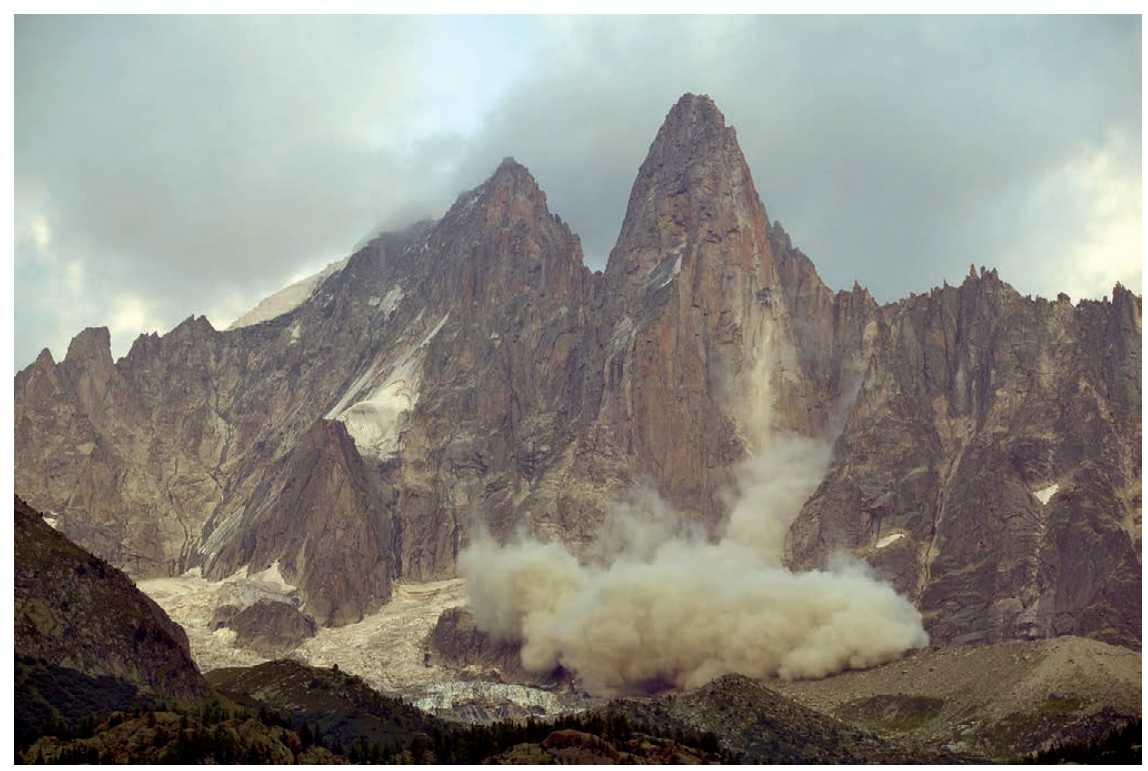

Figure 6. Écroulement dans la face ouest du Petit Dru le 8 août 2003 (photo : Jean-François Hagenmuller). À noter l'aspect très sec de la face nord de l'aiguille Verte (à gauche), qui présente d'ordinaire une large couverture glacio-nivale. 
et al., 2013) rocheux se fonde sur la documentation précise des écroulements - comme dans le massif du MontBlanc où les écroulements sont recensés depuis 2007 au moyen un réseau d'observateurs (Ravanel et al., 2017) -, mais également sur la connaissance de la répartition spatiale du permafrost. Les difficultés de sa cartographie dans les parois tiennent à son invisibilité et son extrême sensibilité aux conditions locales, ce qui rend sa modélisation indispensable. Magnin et al. (2015) ont appliqué au massif du Mont-Blanc un modèle statistique élaboré pour cartographier le permafrost à l'échelle des Alpes, en intégrant des variables d'entrée locales à haute résolution spatiale. Une cartographie du permafrost des parois a ainsi été développée à partir d'un indice permettant d'estimer sa présence (figure 7). Il couvrirait entre 45 et $79 \%$ des $86 \mathrm{~km}^{2}$ de parois (pentes supérieures ou égales à $40^{\circ}$ ) du massif, selon les conditions structurales locales (caractéristiques de la fracturation et de la microtopographie). En effet, l'interprétation de la carte d'indice de permafrost nécessite de tenir compte de l'effet de facteurs autres que le contrôle topoclimatique et qui ne sont pas intégrés au modèle, telles que la fracturation et la présence de neige. Dans des secteurs structuralement favorables (très fracturés), le permafrost pourrait être présent localement dès $1900 \mathrm{~m}$ d'altitude en face nord et $2400 \mathrm{~m}$ en face sud. Il serait plus largement répandu à partir de $2600 \mathrm{~m}$ en face nord et $3000 \mathrm{~m}$ en face sud, mais il ne serait présent dans la totalité des parois qu'à partir de $3600 \mathrm{~m}$, quelle que soit l'exposition.

Sur la base de cette modélisation de la répartition de la température à la surface des parois, l'évolution à long terme du permafrost, d'aujourd'hui à 2100, a été modélisée dans des parois situées entre 3160 et $4300 \mathrm{~m}$ d'altitude (Magnin et al., 2017 ; figure 7). Ces simulations ont été contraintes avec des séries temporelles de température de l'air et pour différents scénarios climatiques (RCP 8.5 et RCP 4.5). Le modèle $2 \mathrm{D}$ à éléments finis de ces parois tient compte de la conduction thermique et des transferts de chaleur latente, et ses sorties pour la période actuelle (2010-2015) ont été évaluées à l'aide des mesures de température dans des forages de $10 \mathrm{~m}$ de profondeur instrumentés depuis 2010 au sommet de l'aiguille du Midi. Il apparaît que les conditions de maintien du permafrost simulées représentent remarquablement bien celles mesurées dans les forages. Au cours des deux

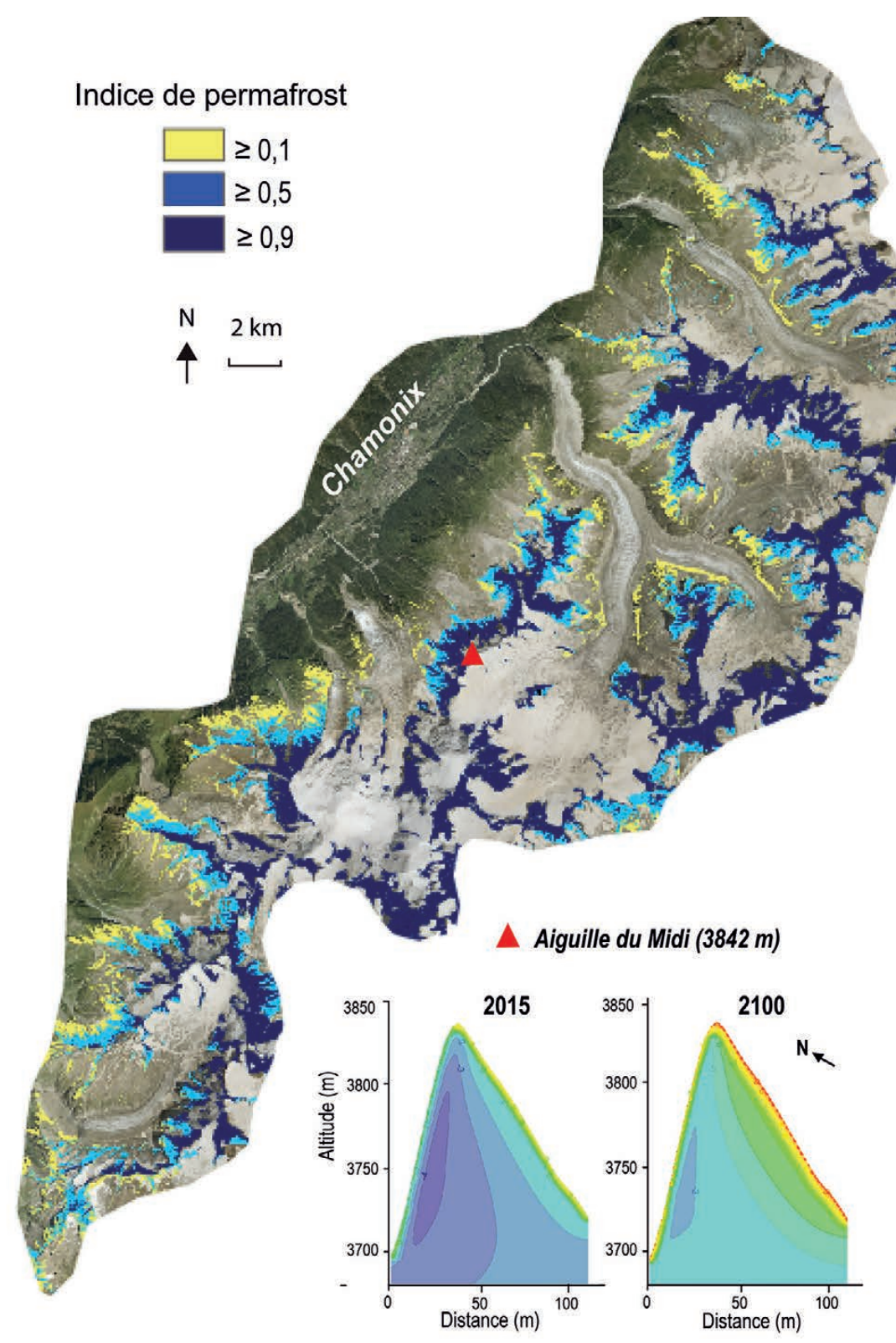

Figure 7. Carte d'indice de permafrost pour les parois du massif du Mont-Blanc et conditions $d u$ permafrost (jusqu'à $-4{ }^{\circ} \mathrm{C}$ pour le violet foncé ; les couleurs chaudes correspondent à des températures positives) à l'aiguille du Midi en 2015 et à la fin du XXle siècle pour un scénario médian (RCP 4.5 ; émissions de gaz à effet de serre limitées). L'indice 0,1 correspond à une température annuelle moyenne à la surface des parois de $0{ }^{\circ} \mathrm{C}+1,28 \sigma$, l'indice 0,5 à une température annuelle moyenne à la surface des parois (TAMSP) $\leq 0^{\circ} \mathrm{C}$, et l'indice 0,9 à une TAMSP de $0{ }^{\circ} \mathrm{C}-1,28 \sigma$. Même avec des températures de surface annuelles moyennes devenant positives, l'inertie thermique de la roche permet la conservation d'un permafrost en profondeur, mais avec un fort déséquilibre thermique.

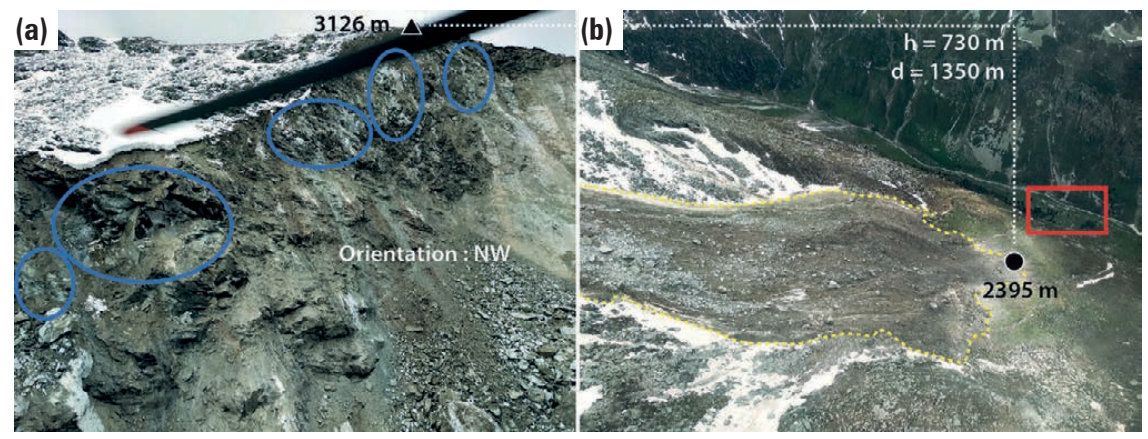

Figure 8. L'écroulement de la Roche d'Étache du 18 juin 2020 (photo : Sylvain Berger). (a) Cicatrice haute de plusieurs dizaines de mètres ; les cercles bleus indiquent la présence de glace massive. (b) Partie basse ( 700 derniers mètres) du dépôt ; cadre rouge : chalets de Saint-Barthélemy (2 $020 \mathrm{~m})$; h : dénivellation entre le sommet de la cicatrice et le front du dépôt ; $d$ : distance de parcours horizontale. 
dernières décennies, le permafrost a disparu des versants sud au-dessous de $3300 \mathrm{~m}$, voire plus à l'amont. Le permafrost tempéré (au point de fusion de l'eau dont la température - proche de $0{ }^{\circ} \mathrm{C}$ - varie en fonction des impuretés et de la pression) s'est étendu jusqu'à 3300 et $3850 \mathrm{~m}$ dans les faces nord et sud, respectivement. Au cours du $\mathrm{XXI}^{\mathrm{e}}$ siècle, ce permafrost tempéré devrait s'étendre au moins jusqu'à $4300 \mathrm{~m}$ d'altitude sur les parois rocheuses exposées au sud et jusqu'à $3850 \mathrm{~m}$ sur les faces nord. Dans le cas le plus pessimiste et malgré une diffusion de la chaleur très lente en profondeur, les conditions de permafrost disparaîtraient de la surface des versants sud au-dessous de $4300 \mathrm{~m}$, tandis que le permafrost tempéré atteindrait $3850 \mathrm{~m}$ dans les versants nord.

\section{Conclusions et pistes de recherche}

Les projections climatiques pour le $\mathrm{XXI}^{\mathrm{e}}$ siècle suggèrent :

i) une accélération du réchauffement. Les deux nouveaux modèles français qui participent au programme mondial de simulations du climat (CMIP6) prévoient un réchauffement plus élevé à l'horizon 2100 que les modèles établis en 2012, en particulier pour les scénarios les plus pessimistes en émissions. Selon le scénario le plus « pessimiste » (SSP5 8,5 - croissance économique rapide alimentée par des énergies fossiles), l'augmentation de la température moyenne globale atteindrait 6 à $7^{\circ} \mathrm{C}$ en 2100 , soit $1^{\circ} \mathrm{C}$ de plus que dans les précédentes simulations ; ii) une modification du cycle annuel des précipitations avec une diminution des cumuls estivaux et une légère augmentation des cumuls hivernaux ; iii) des changements dans les extrêmes climatiques avec un raccourcissement des périodes de retour des précipitations extrêmes, notamment liquides. Or, l'eau liquide qui pénètre dans les fractures de la roche augmente la pression hydrostatique et donc le risque de déstabilisation des parois.

Ces évolutions climatiques auront un effet majeur sur la cryosphère alpine et notamment sur la dégradation du permafrost. Aussi peut-on s'attendre à une poursuite et même très vraisemblablement à une accélération de la déstabilisation des parois rocheuses de haute montagne, avec non seulement une augmentation de la fréquence des écroulements - comme on l'a montré pour la période récente -, mais également de leur volume - comme suggéré par de grands événements récents comme celui du Piz Cengalo. C'est là une mauvaise nouvelle pour une partie des infrastructures de haute montagne des Alpes françaises, dont
947 éléments sont construits entre 2430 et 4363 m d'altitude sur des terrains caractérisés par un permafrost modélisé comme probable. C'est également une mauvaise nouvelle pour les alpinistes dont le terrain de jeu se réduit au regard de l'évolution des parois et de leur dangerosité croissante.

Pour mieux comprendre les processus de déstabilisation des parois rocheuses à permafrost de haute montagne, il est nécessaire : i) d'améliorer la modélisation thermique des parois en effectuant une descente d'échelle des résultats des modèles climatiques à l'échelle locale afin de mieux prendre en compte les effets topographiques tout en simulant les processus de transfert de chaleur en 3D, ii) d'avancer dans la modélisation des processus thermo-hydro-mécaniques à l'origine de la déstabilisation des parois, et iii) de poursuivre l'analyse des écroulements recensés dans le massif du Mont-Blanc et ailleurs dans les Alpes, comme celui du 18 juin 2020 dans le vallon d'Étache (Haute Maurienne ; figure 8) dont le volume pourrait avoisiner $300000 \mathrm{~m}^{3}$. Si la glace massive qui occupait une superficie notable de la cicatrice suggère un rôle important du permafrost dans le déclenchement, la date d'occurrence en tout début de saison estivale est plus surprenante, probablement associée à l'infiltration et au regel de l'eau de fonte de la neige.

\section{Bibliographie}

Bozonnet R., Bravard Y., Chardon M., 1983. Le Mont-Blanc. Coll. Trésors de la Savoie, 288 p.

Deline P., 2009. Interactions between rock avalanches and glaciers in the Mont-Blanc massif during the late Holocene. Quat. Sci. Rev., 28, 1070-1083.

Gallach X., Carcaillet J., Ravanel L., Deline P., Ogier C., Rossi M., Malet E., Garcia-Sellés D., 2020. Climatic and structural controls on Lateglacial and Holocene rockfall occurrence in high-elevated rock walls of the Mont-Blanc massif (Western Alps). Earth Surface Processes and Landforms. doi: 10.1002/esp.4952

Gruber S., Haeberli W., 2007. Permafrost in steep bedrock slopes and its temperature-related destabilization following climate change. J. Geophys. Res., 112, F00547.

Gruber S., Hoelzle M., Haeberli W., 2004. Permafrost thaw and destabilization of Alpine rock walls in the hot summer of 2003. Geophys. Res. Lett., 31, L13504. doi: 10.1029/2004GL020051

Haeberli W., Wegmann M., Vonder Mühll D., 1997. Slope stability problems related to glacier shrinkage and permafrost degradation in the Alps. Eclogae Geologicae Helvetiae, $90,407-414$

Krautblatter M., Funk D., Günzel F.K., 2013. Why permafrost rocks become unstable: a rock-ice-mechanical model in time and space. Earth Surface Processes and Landforms, 38, 876-887

Magnin F., Brenning A., Bodin X., Deline P., Ravanel L., 2015. Statistical modelling of rock wall permafrost distribution: application to the Mont-Blanc massif. Géomorphologie, $21,145-162$

Magnin F., Josnin J.-Y., Ravanel L., Pergaud J., Pohl B., Deline P., 2017. Modelling rock wall permafrost degradation in the Mont-Blanc massif from the LIA to the end of the 21 st century. The Cryosphere, 11, 1813-1834.

Ravanel L., Deline P., 2008. La face ouest des Drus (massif du Mont-Blanc) : évolution de l'instabilité d'une paroi rocheuse dans la haute montagne alpine depuis la fin du Petit Age Glaciaire. Géomorphologie, 4, 261-272.

Ravanel L., Deline P., 2011. Climate influence on rockfalls in high-Alpine steep rockwalls: the North side of the Aiguilles de Chamonix (Mont Blanc massif) since the end of the Little Ice Age. The Holocene, 21, 357-365.

Ravanel L., Magnin F., Deline P., 2017. Impacts of the 2003 and 2015 summer heat waves on permafrost-affected rockwalls in the Mont Blanc massif. Science of the Total Environment, 609, 132-143. 